\title{
Expanding the gelation properties of valine-based 3,5-diaminobenzoate organogelators with $\mathbf{N}$-alkylurea functionalities
}

\author{
Hak-Fun Chow ${ }^{* 1,2,3}$ and Chin-Ho Cheng ${ }^{1}$
}

\section{Full Research Paper}

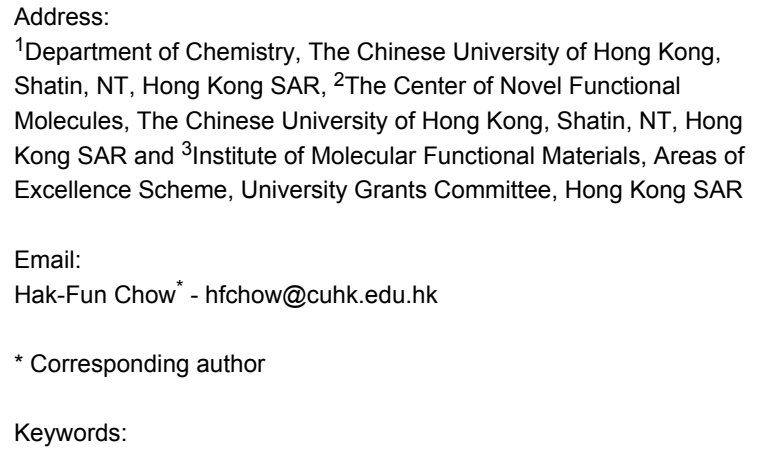

amino acids; hydrophobic effect; organogelators; self-assembly; urea
Beilstein J. Org. Chem. 2010, 6, 1015-1021. doi:10.3762/bjoc.6.114

Received: 29 June 2010

Accepted: 12 October 2010

Published: 26 October 2010

Guest Editor: J.-P. Desvergne

(c) 2010 Chow and Cheng; licensee Beilstein-Institut. License and terms: see end of document.

\begin{abstract}
A new family of valine-containing 3,5-diaminobenzoate derivatives 2 with $N$-alkylurea moieties attached to the valine moieties was prepared. By appending these two new $N$-alkylurea chains to the molecular structure, their organogelating properties were extended from only aromatic solvents, to a wide range of other types of solvents such as alicyclic hydrocarbons, alcohols and polar solvents such as DMSO and DMF. It was also found that a longer $N$-alkylurea chain conferred improved gelation power and higher thermal stability as compared to those of the shorter ones.
\end{abstract}

\section{Introduction}

Organic gelators are an interesting group of molecules that are able to form a non-covalent three dimensional network with a particular solvent system. The ultimate result is the immobilization of solvent molecules. These molecules possess many potential applications in biomedical science, environmental and separation technology [1-6].

One key problem in the design and synthesis of organogelators lies in the difficulty in predicting their gelation properties beforehand. Most often a subtle change of the substituents of the organgelator can lead to substantial modification in its gelating properties. This is because gelation is the result of a delicate balance of various driving forces. If the binding interactions between the organogelator molecules are too strong, precipitation or crystallization will take place. On the other hand, if the interactions are too weak, dissolution of the organogelator will result. In addition, non-specific interactions such as hydrophobic and van der Waals interactions are diffi- 
<smiles>[R]C(NC(=O)OCc1ccccc1)C(=O)Nc1cc(NC(=O)[N+](=O)[O-])cc(C(=O)OCc2ccccc2)c1</smiles>

1

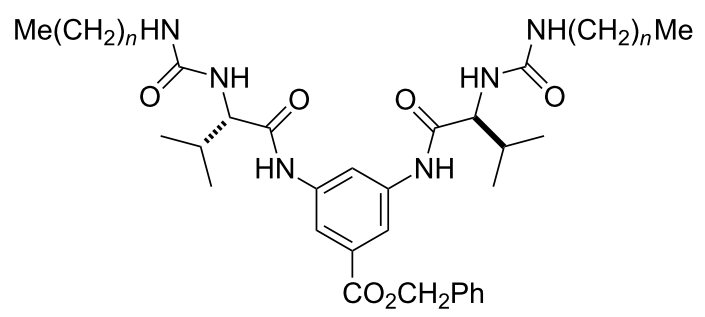

2

Figure 1: Amino acid based organogelators 1 and 2.

cult to quantify, yet they can play very important roles on a cumulative scale when such interactions actually involve a large ensemble of solvent and gelator molecules.

Our group has been interested in the synthesis and self-assembled gelating properties of amino acid-containing non-dendritic $[7,8]$ and dendritic molecules $[9,10]$. We recently showed that the organogelation strength in aromatic solvents of a series of $\alpha$-amino acid-based low molecular weight organogelators 1 (Figure 1) could be enhanced by appending additional aromaticcontaining substituents [7]. Both the Cbz protecting group and the benzyl ester functionality were responsible for the enhanced gelating power in aromatic solvents. However, they are poor organogelators in non-aromatic solvents such as alkanes, alcohols, acetone, acetonitrile and DMSO. In order to further expand their gelating power in other solvents, we decided to further modify the appending $\mathrm{Cbz}$ groups with other functionalities. One particular interesting moiety is the urea group, which can act simultaneously as a donor and an acceptor of hydrogen bonds and is known to be a key structural element in many organogelators [11-14]. Herein we report that the organogelating properties of 1 could be expanded to include alicyclic hydrocarbon, alcohols and even polar aprotic solvents such as DMSO and DMF by replacing the Cbz group with an $\mathrm{N}$-alkylurea functionality (e.g., 2). In addition, the length of the alkyl chain $-\left(\mathrm{CH}_{2}\right)_{n} \mathrm{Me}$ also exhibited some interesting effects on their gelation ability [15].

\section{Results and Discussion Synthesis}

In our earlier report it was shown that, amongst the many $\alpha$-amino acids used, valine-based $\mathbf{1}\left(\mathrm{R}=\mathrm{CHMe}_{2}\right)$ possessed much better organogelating properties. Hence in the present study we focused only on valine derivatives 2 . A homologous series of $N$-alkylurea side chain derivatives 2 ( $n=3-6,9,10$, $12,15,18$ and 20) was prepared in order to evaluate effect of the length of the alkyl side chain on the resulting gelation properties.

The target organogelators were prepared according to Scheme 1. The known Boc protected benzyl ester 3 [7] was converted into the corresponding diamino compound $\mathbf{4}$ in $98 \%$ yield by treatment with trifluoroacetic acid (TFA) followed by neutralization with $\mathrm{NaHCO}_{3}$. The $\mathrm{N}$-alkylurea derivatives 2 were then obtained in $88-94 \%$ as white solids by coupling compound 4 with various $O$-succinimidyl alkylcarbamates 6 ,

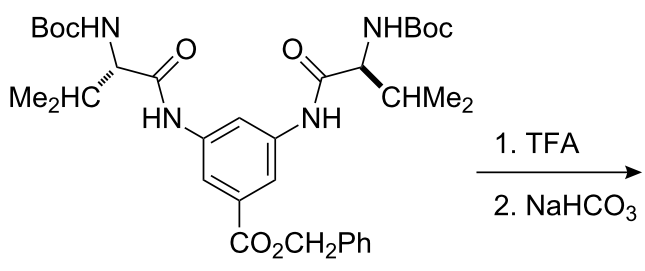

3<smiles>CC(N)C(=O)Nc1cc(NC(=O)C(N)C(C)C)cc(C(=O)OCc2ccccc2)c1</smiles>

4

1. EtOCOCl, $N$-methylmorpholine

$$
\begin{array}{cl}
\mathrm{Me}\left(\mathrm{CH}_{2}\right)_{n} \mathrm{CO}_{2} \mathrm{H} & \stackrel{\text { 2. } \mathrm{NaN}_{3}}{\text { 3. toluene, } 65^{\circ} \mathrm{C}} \\
5 & \text { 4. N-hydroxysuccinimide, pyridine }
\end{array}
$$


prepared from the corresponding alkanoic acids $\mathbf{5}$ according to a literature procedure [16], in the presence of $N$-diisopropylethylamine.

\section{Structural characterization}

All synthesized compounds were characterized by ${ }^{1} \mathrm{H}$ and ${ }^{13} \mathrm{C}$ nuclear magnetic resonance spectroscopy, mass spectrometry, elemental analysis, and optical polarimetry. Due to the poor solubilities of the target organogelators 2 in chloroform and acetone, their ${ }^{1} \mathrm{H}$ and ${ }^{13} \mathrm{C}$ NMR spectra were recorded in DMSO- $d_{6}$. In addition, the ${ }^{1} \mathrm{H}$ and ${ }^{13} \mathrm{C}$ NMR spectra of analogues with thirteen or more carbon atoms in the aliphatic hydrocarbon chains were recorded at $100{ }^{\circ} \mathrm{C}$ to avoid gelation of the solvent. The ${ }^{1} \mathrm{H}$ NMR spectra of compounds 2 showed the presence of the aliphatic hydrocarbon chains and the urea moieties. The methyl group of the aliphatic chains appeared as a triplet at $\sim \delta 0.86$, while the methylenes of the aliphatic chains were located in close proximity to the signals of the valine side chain. On the other hand, the signals from the isopropyl group of the valine residue overlapped with those of the methylenes of the aliphatic chains. The two NH protons of the urea groups were different and appeared as a doublet at $\delta 6.04$ and a triplet at $\delta 6.07$. The benzyl ester group appeared as a singlet at $\delta 5.24$ and a set of multiplets at $\delta 7.35-7.47$. Finally, the aromatic protons of the 3,5-diaminobenzoate moiety appeared as two singlets at $\delta 7.98$ and $\delta 8.26$.

The ${ }^{13} \mathrm{C}$ NMR signals of the target molecules 2 could be grouped into three separate regions. First, in the downfield region, the ${ }^{13} \mathrm{C}$ signals located at $\delta 157,165$ and 171 were attributed to the urea, ester and anilide $\mathrm{C}=\mathrm{O}$ groups, respectively. Second, the ${ }^{13} \mathrm{C}$ signals of the central 3,5-diamino-substituted aromatic core were scattered between $\delta$ 110-140, while the aromatic signals due to the benzyl ester were found at $\sim \delta$ 127-135. Finally, the ${ }^{13} \mathrm{C}$ signals of the valine side chain and the aliphatic hydrocarbon side chain were located in a range of $\delta 12-66$. Specifically, the ${ }^{13} \mathrm{C}$ signal situated at $\delta 58$ corresponded to the valine $\alpha$-carbon. The benzylic carbon appeared at $\delta$ 66. The remaining aliphatic ${ }^{13} \mathrm{C}$ signals due to the aliphatic carbons and some of the resonance peaks overlapped with each other. Hence, the number of the observed signals was sometimes less than theoretically predicted. One ${ }^{13} \mathrm{C}$ signal $\left(\mathrm{CH}_{2} \mathrm{NH}\right)$ was located at $\delta 39$ and was often buried within the residual solvent signals of DMSO- $d_{6}$. For organogelators with longer aliphatic chains, this signal was too weak to be observed and assignment could only be made based on the chemical shift value of other homologues where this signal could be observed.

High-resolution mass spectra were recorded for all synthesized compounds. The experimental determined $\mathrm{M}^{+}$results were in accordance with the theoretical values. Generally, the abun- dance of the $\mathrm{M}^{+}$decreased with increasing length of the alkyl hydrocarbon side chain.

\section{Gelation properties}

The gelation behavior of bis( $N$-alkylurea valine) benzyl esters 2 were examined at a concentration of $2 \% \mathrm{w} / \mathrm{v}$ in various solvents (Table 1). Most organogelators 2 were found to be insoluble in common non-aromatic organic solvents at room temperature. In contrast, they were soluble in aromatic solvents and organic solvents of high polarity such as 1,4-dioxane, DMF and DMSO upon warming. Interestingly, homologues having shorter aliphatic chain $(n<10)$ were poor organogelators, while the longer aliphatic chain analogues $(n \geq 10)$ formed transparent gels in aromatic solvents and translucent gels in 1,4-dioxane, DMF and DMSO. Most interestingly, it was observed that organogelator $2(n=20)$ with the longest hydrocarbon chain also formed opaque gels in alcoholic solvents and translucent gels in alicyclic hydrocarbon solvents, respectively. Hence, the range of the solvents that these new compounds can gel was significantly expanded by replacing the $\mathrm{Cbz}$ group with an $\mathrm{N}$-alkylurea functionality.

Based on these experiments, organogelators 2 with longer aliphatic hydrocarbon chains $(n \geq 10)$ were found to exhibit better gelation behavior in aromatic solvents. However, it was interesting to note that compounds with less than or equal to eight carbon atoms in the side chain failed to induce gelation. The effect of aliphatic hydrocarbon chain length $(n=10,12,15$, 18 and 20) on their gelation power was then compared by the determination of their minimum gelation concentration (MGC) and gel-sol transition temperature $\left(T_{\mathrm{g}}\right)$ in different solvents (Table 2). It was found that the longer the alkyl chain, the lower the MGC but the higher the $T_{\mathrm{g}}$ value. Hence, the cumulative hydrophobic interaction between organogelator molecules with longer aliphatic chains must be stronger than that of the shorter ones, and this factor should contribute to the higher thermal stability of the longer chain organogels. In addition, the presence of the long aliphatic chain also prevented the organogelators from crystallizing by imposing a higher degree of local disorder.

Fourier transform infrared spectroscopy (FT-IR) was employed to elucidate the extent of intermolecular hydrogen bonding in the different macroscopic phases of organogelator $2(n=20)$ in $o$-xylene (Table 3 ). In $1 \% \mathrm{w} / \mathrm{v}$ hot $o$-xylene solution $\left(100^{\circ} \mathrm{C}\right)$, two peaks at 3342 and $3247 \mathrm{~cm}^{-1}$ in the $v_{\mathrm{N}-\mathrm{H}}$ regions were identified. These two signals could be assigned to the stretching bands of urea $\mathrm{N}-\mathrm{H}$ and anilide $\mathrm{N}-\mathrm{H}$, respectively. The two peaks at 1729 and $1629 \mathrm{~cm}^{-1}$ in the $v_{\mathrm{C}=\mathrm{O}}$ region were found and they were attributed to the anilide $\mathrm{C}=\mathrm{O}$ and urea $\mathrm{C}=\mathrm{O}$, respectively. Signal assignments of the urea $\mathrm{C}=\mathrm{O}$ and $\mathrm{N}-\mathrm{H}$ were 
Table 1: Gelation behavior of bis( $N$-alkylurea valine) compounds 2 at $2 \% \mathrm{w} / \mathrm{v}^{\mathrm{a}}$

\begin{tabular}{|c|c|c|c|c|c|c|c|}
\hline Solvent & $2(n=3-6)$ & $2(n=9)$ & $2(n=10)$ & $2(n=12)$ & $2(n=15)$ & $2(n=18)$ & $2(n=20)$ \\
\hline THF & I & $\mathrm{P}$ & $\mathrm{P}$ & $\mathrm{P}$ & $\mathrm{P}$ & $P$ & $P$ \\
\hline acetone & I & I & I & I & I & I & I \\
\hline chloroform & I & I & 1 & I & I & 1 & 1 \\
\hline dichloromethane & I & 1 & 1 & I & I & 1 & 1 \\
\hline ethyl acetate & I & I & I & I & I & 1 & I \\
\hline ethylene glycol & 1 & 1 & 1 & 1 & 1 & 1 & 1 \\
\hline 1-butanol & - & - & - & - & - & - & OG \\
\hline 1-heptanol & - & - & - & - & - & - & OG \\
\hline 1-dodecanol & - & - & - & - & - & - & OG \\
\hline cyclooctanol & - & - & - & - & - & - & TG \\
\hline hexane & 1 & 1 & 1 & I & 1 & 1 & 1 \\
\hline cyclohexene & - & - & - & - & - & - & TG \\
\hline cyclooctene & - & - & - & - & - & - & TG \\
\hline 1,4-dioxane & $S$ & $S$ & $S$ & TG & TG & TG & TG \\
\hline DMF & $S$ & $\mathrm{~s}$ & $\mathrm{~s}$ & TG & TG & TG & TG \\
\hline DMSO & $\mathrm{s}$ & $\mathrm{s}$ & TG & TG & TG & TG & TG \\
\hline anisole & S & S & CG & CG & CG & CG & CG \\
\hline benzene & $S$ & $S$ & $\mathrm{~S}$ & CG & CG & CG & CG \\
\hline benzyl alcohol & $S$ & CG & CG & CG & CG & CG & CG \\
\hline o-dichlorobenzene & $S$ & CG & CG & CG & CG & CG & CG \\
\hline nitrobenzene & $S$ & $S$ & TG & TG & TG & TG & TG \\
\hline toluene & $S$ & CG & CG & CG & CG & CG & CG \\
\hline o-xylene & $S$ & CG & CG & CG & CG & CG & CG \\
\hline
\end{tabular}

a = insoluble; $\mathrm{P}$ = precipitation; $\mathrm{S}=$ soluble; $\mathrm{CG}$ = clear transparent gel; $\mathrm{OG}=$ opaque gel; $\mathrm{TG}=$ translucent gel.

based on the spectral data of a model compound, namely 1,3didodecylurea. In the FT-IR spectrum of $2(n=20)(1 \% \mathrm{w} / \mathrm{v})$ in $o$-xylene gel at $25{ }^{\circ} \mathrm{C}$, the corresponding peaks were identified at $3335,3268,1728$ and $1628 \mathrm{~cm}^{-1}$, respectively. Hence, there was little difference in terms of the stretching frequencies both in solution and in the gel state. Furthermore, both sets of values are very similar to those of $\mathbf{2}(n=20)$ in solid $\mathrm{KBr}$, where the $\mathrm{C}=\mathrm{O}$ and $\mathrm{N}-\mathrm{H}$ moieties are known to form extensive intermolecular hydrogen bonds. Hence, it is very likely that compound $\mathbf{2}$ exists as aggregates via intermolecular hydrogen bonding in solution state due to the extremely strong hydrogen bonding property of the urea moiety. In addition, broadening of absorption signals in the gel state was observed which suggests that further intermolecular hydrogen bonding occurred during gel formation.

\section{Conclusion}

We have reported the synthesis of novel valine-containing 3,5diaminobenzoate derivatives $\mathbf{2}$ with additional $N$-alkylurea functionality at the $N$-terminal of the valine residues. The resulting organogelators were found to possess gelating ability that covered a wider range of organic solvents, including alco-

Table 2: Minimum gelation concentration and gel-to-sol transition temperature of compounds $\mathbf{2}(n=10,12,15,18$ and 20$)$.

\begin{tabular}{|c|c|c|c|c|c|c|c|c|c|c|}
\hline \multirow[t]{2}{*}{ Solvent } & \multicolumn{2}{|c|}{$2(n=10)$} & \multicolumn{2}{|c|}{$2(n=12)$} & \multicolumn{2}{|c|}{$2(n=15)$} & \multicolumn{2}{|c|}{$2(n=18)$} & \multicolumn{2}{|c|}{$2(n=20)$} \\
\hline & $\mathrm{MGC}^{\mathrm{a}}$ & $T_{\mathrm{g}}^{\mathrm{b}}$ & MGC & $T_{\mathrm{g}}$ & MGC & $T_{\mathrm{g}}$ & MGC & $T_{\mathrm{g}}$ & MGC & $T_{\mathrm{g}}$ \\
\hline benzyl alcohol & 1.8 & - & 1.8 & 70 & 1.5 & 79 & 1.2 & 88 & 1.0 & 94 \\
\hline o-dichlorobenzene & 1.5 & - & 1.2 & 109 & 1.3 & 113 & 1.0 & 116 & 0.8 & 120 \\
\hline o-xylene & 1.6 & - & 1.5 & 75 & 1.5 & 80 & 1.0 & 83 & 1.0 & 86 \\
\hline
\end{tabular}

an w/v\%; In ${ }^{\circ} \mathrm{C}$ as determined by inverted tube method. 


\begin{tabular}{lllr|}
\hline Table 3: FT-IR data of compound $\mathbf{2}(n=20)$ and 1,3-di(dodecyl)urea ${ }^{\mathrm{a}}$. & & anilide $\mathrm{N}-\mathrm{H}$ & anilide $\mathrm{C}=\mathrm{O}$ \\
Samples & urea $\mathrm{N}-\mathrm{H}$ & 3247 & 1729 \\
\hline $\mathbf{2}(n=20)$ in 0 -xylene solution at $100^{\circ} \mathrm{C}$ & 3342 & 3268 & 1728 \\
$\mathbf{2}(n=20)$ in 0 -xylene gel at $25^{\circ} \mathrm{C}$ & 3335 & 3248 & 1730 \\
$\mathbf{2}(n=20)$ in solid in $\mathrm{KBr}$ pellet & 3348 & - & 1629 \\
$1,3-\mathrm{di}($ dodecyl)urea in solid $\mathrm{KBr}$ pellet & 3340 & - & 1622 \\
\hline
\end{tabular}
a $\mathrm{cm}^{-1}$.

holic, aromatic, alicyclic hydrocarbon and polar solvents. Furthermore, attachment of longer aliphatic chains not only enhanced hydrophobic interactions between the organogelators, but also prevented crystallization of the self assembled aggregates during gelation, producing gels with a higher $T_{\mathrm{g}}$ and a lower MGC value.

\section{Experimental}

General. Optical rotation measurements were conducted in DMSO (unless otherwise stated) and below the MGC to avoid molecular aggregation or gelation and were measured with a Perkin Elmer 341 polarimeter. The starting materials 6 were prepared according to a literature procedure [16]. General procedures, yields and characterization data of compounds $6(n=$ 3-20) can be found in Supporting Information File 1.

Compound 4. Trifluoroacetic acid $(50 \mathrm{~mL}, 60 \mathrm{mmol})$ was added to a solution of the benzyl ester 3 [2] (6.40 g, $10.0 \mathrm{~mol})$ in $\mathrm{CH}_{2} \mathrm{Cl}_{2}(100 \mathrm{~mL})$ at $25^{\circ} \mathrm{C}$. The progress of deprotection was monitored by TLC. Upon complete deprotection $(\sim 12 \mathrm{~h})$, the solvent was removed on a rotary evaporator. The crude product was made alkaline by the addition of aqueous $\mathrm{NaHCO}_{3}$ solution to $\mathrm{pH}$ 8. The mixture was extracted with $\mathrm{CH}_{2} \mathrm{Cl}_{2}$ $(50 \mathrm{~mL} \times 3)$, the combined organic layers were washed with saturated $\mathrm{NaCl}$ solution $(100 \mathrm{~mL} \times 2)$, dried $\left(\mathrm{MgSO}_{4}\right)$, filtered and concentrated in vacuo to give compound $\mathbf{4}$ as a pale yellow liquid $(4.3 \mathrm{~g}, 98 \%) .[\alpha]_{\mathrm{D}}{ }^{20}-177.6\left(c 0.50, \mathrm{CHCl}_{3}\right) .{ }^{1} \mathrm{H} \mathrm{NMR}$ (DMSO- $\left.d_{6}\right): \delta 0.85\left(6 \mathrm{H}, \mathrm{d}, J=6.9, \mathrm{CH}\left(\mathrm{CH}_{3}\right) \mathrm{Me}\right), 0.91(6 \mathrm{H}, \mathrm{d}$, $\left.J=6.9, \mathrm{CH}(\mathrm{Me}) \mathrm{CH}_{3}\right), 1.85-1.99\left(2 \mathrm{H}, \mathrm{m}, \mathrm{CH}\left(\mathrm{CH}_{3}\right)_{2}\right), 3.12(2 \mathrm{H}$, $\left.\mathrm{d}, J=5.7, \mathrm{H}_{2} \mathrm{NCHCH}\right), 3.2-3.5(6 \mathrm{H}$, brs, $\mathrm{NH}), 5.35(2 \mathrm{H}, \mathrm{s}$, $\left.\mathrm{PhCH}_{2}\right), 7.36-7.49$ (5H, m, $\left.\mathrm{Ar} H\right), 7.98(2 \mathrm{H}, \mathrm{d}, J=1.8, \mathrm{Ar} H)$, $8.35(1 \mathrm{H}, \mathrm{t}, J=1.8, \mathrm{Ar} H) .{ }^{13} \mathrm{C}$ NMR (DMSO- $\left.d_{6}\right): \delta 17.4,19.5$, $31.8,60.8,66.4,114.4,114.7,128.2,128.4,128.6,130.3,136.1$, 139.6, 165.4, 174.1. MS (FAB): $441\left(\mathrm{M}+\mathrm{H}^{+}, 30 \%\right)$. HRMS (LSIMS): calcd for $\mathrm{C}_{24} \mathrm{H}_{32} \mathrm{~N}_{4} \mathrm{O}_{4}, 441.2496$; found, 441.2505. Anal. found: $\mathrm{C}, 65.63 ; \mathrm{H}, 7.50 ; \mathrm{N}, 12.71 . \mathrm{C}_{24} \mathrm{H}_{32} \mathrm{~N}_{4} \mathrm{O}_{4}$ requires C, 65.43; H, 7.32; N, 12.71 .

General procedure for the preparation of bis- $(\mathrm{N}$-alkylurea) benzyl esters 2 . A mixture of $O$-succinimidyl carbamate 6 (10.0 mmol) and diisopropylethylamine $(1.8 \mathrm{~mL}, 10.0 \mathrm{mmol})$ was added to a THF solution $(100 \mathrm{~mL})$ of the diamino benzyl ester 4 ( $2.2 \mathrm{~g}, 5.0 \mathrm{mmol})$. The reaction mixture was stirred at 25 ${ }^{\circ} \mathrm{C}$ for $4 \mathrm{~h}$. The insoluble crude solid product was filtered and washed successively with boiling $n$-hexane $(100 \mathrm{~mL})$, acetone $(100 \mathrm{~mL})$ and THF $(100 \mathrm{~mL})$ to afford the title compound.

$2(n=3)$. The product was obtained as a white solid (3.0 g, 94\%) from $O$-succinimidyl butylcarbamate $6(n=3)(2.2 \mathrm{~g}$, $10.0 \mathrm{mmol}) .[\alpha]_{\mathrm{D}}=+62.4$ (c 1.01). mp 221-222 ${ }^{\circ} \mathrm{C} .{ }^{1} \mathrm{H}$ NMR $\left(\mathrm{DMSO}-d_{6}\right): \delta \quad 0.83-0.90\left(18 \mathrm{H}, \mathrm{m}, \mathrm{CH}\left(\mathrm{CH}_{3}\right)_{2}\right.$ and $\left.\mathrm{CH}_{2} \mathrm{CH}_{2} \mathrm{CH}_{3}\right), 1.22-1.36(8 \mathrm{H}, \mathrm{m}$, aliphatic $H), 1.92-1.94(2 \mathrm{H}$, $\left.\mathrm{m}, \mathrm{CHCH}\left(\mathrm{CH}_{3}\right)_{2}\right), 2.99\left(4 \mathrm{H}, \mathrm{q}, \mathrm{J}=6, \mathrm{NHCH}_{2} \mathrm{CH}_{2}\right), 4.18(2 \mathrm{H}$, $\mathrm{dd}, J=8.7$ and 6.6, $\mathrm{NHCHCH}), 5.34\left(2 \mathrm{H}, \mathrm{s}, \mathrm{PhCH}_{2}\right), 6.05(2 \mathrm{H}$, $\mathrm{d}, J=4.2$, urea $\mathrm{N} H), 6.09(2 \mathrm{H}, \mathrm{t}, J=5.6$, urea $\mathrm{N} H), 7.35-7.47$ $(5 \mathrm{H}, \mathrm{m}, \operatorname{Ar} H), 7.99(2 \mathrm{H}, \mathrm{s}, \operatorname{Ar} H), 8.28(1 \mathrm{H}, \mathrm{s}, \mathrm{Ar} H), 10.30(2 \mathrm{H}$, s, CONHAr). ${ }^{13} \mathrm{C}$ NMR (DMSO- $d_{6}$ ): $\delta 13.7,17.9,19.3,19.5$, 31.2, 32.1, 38.9, 58.7, 66.4, 114.4, 114.8, 128.2, 128.4, 128.6, 130.3, 136.0, 139.6, 157.9, 165.4, 171.8. MS (FAB) 639 (M + $\mathrm{H}^{+}, 12 \%$ ). HRMS (LSIMS): calcd for $\mathrm{C}_{34} \mathrm{H}_{50} \mathrm{~N}_{6} \mathrm{O}_{6}, 639.3865$; found, 639.3854. Anal. found: C, 63.75; H, 7.95; N, 13.08 . $\mathrm{C}_{34} \mathrm{H}_{50} \mathrm{~N}_{6} \mathrm{O}_{6}$ requires $\mathrm{C}, 63.93 ; \mathrm{H}, 7.89 ; \mathrm{N}, 13.15$.

$2(n=4)$. The compound was obtained as a white solid (3.1 g, 94\%) from $O$-succinimidyl pentylcarbamate $6(n=4)(2.3 \mathrm{~g}$, $10.0 \mathrm{mmol}) .[\alpha]_{\mathrm{D}}=+57.9$ (c 1.08). $\mathrm{mp} 224-225{ }^{\circ} \mathrm{C} .{ }^{1} \mathrm{H}$ NMR $\left(\mathrm{DMSO}-d_{6}\right): \delta$ 0.82-0.90 $\left(18 \mathrm{H}, \mathrm{m}, \mathrm{CH}\left(\mathrm{CH}_{3}\right)_{2}\right.$ and $\left.\mathrm{CH}_{2} \mathrm{CH}_{2} \mathrm{CH}_{3}\right), 1.20-1.39(12 \mathrm{H}, \mathrm{m}$, aliphatic $H), 1.91-1.95$ $\left(2 \mathrm{H}, \mathrm{m}, \mathrm{CHCH}\left(\mathrm{CH}_{3}\right)_{2}\right), 2.97\left(4 \mathrm{H}, \mathrm{q}, J=6, \mathrm{NHCH}_{2} \mathrm{CH}_{2}\right), 4.16$ $(2 \mathrm{H}, \mathrm{dd}, J=9.0$ and 6.6, $\mathrm{NHCHCH}), 5.34\left(2 \mathrm{H}, \mathrm{s}, \mathrm{PhCH}_{2}\right), 6.05$ $(2 \mathrm{H}, \mathrm{d}, J=4.5$, urea $\mathrm{N} H), 6.08(2 \mathrm{H}, \mathrm{t}, J=5.6$, urea $\mathrm{N} H)$, 7.38-7.48 (5H, m, ArH), 7.98 (2H, s, ArH), $8.26(1 \mathrm{H}, \mathrm{s}, \operatorname{Ar} H)$, 10.30 ( $2 \mathrm{H}, \mathrm{s}, \mathrm{CONHAr}) .{ }^{13} \mathrm{C}$ NMR (DMSO- $\left.d_{6}\right): \delta 13.9,17.9$, 19.3, 21.9, 28.6, 29.6, 31.2, 39.2, 58.7, 66.4, 114.4, 114.8, 128.1, 128.2, 128.6, 130.3, 136.0, 139.6, 157.9, 165.3, 171.8. MS (FAB) $667\left(\mathrm{M}+\mathrm{H}^{+}, 10 \%\right)$. HRMS (LSIMS): calcd for $\mathrm{C}_{36} \mathrm{H}_{54} \mathrm{~N}_{6} \mathrm{O}_{6}, 667.4178$; found, 667.4185. Anal. found: $\mathrm{C}$, 64.77; $\mathrm{H}, 8.20 ; \mathrm{N}, 12.53 . \mathrm{C}_{36} \mathrm{H}_{54} \mathrm{~N}_{6} \mathrm{O}_{6}$ requires $\mathrm{C}, 64.84 ; \mathrm{H}$, $8.16 ; \mathrm{N}, 12.60$.

$2(n=5)$. The product was obtained as a white solid (3.2 g, $91 \%)$ from $O$-succinimidyl hexylcarbamate $6(n=5)(2.4 \mathrm{~g}$, 
$10.0 \mathrm{mmol}) .[\alpha]_{\mathrm{D}}=+63.6$ (c 0.99). $\mathrm{mp} 225-226{ }^{\circ} \mathrm{C} .{ }^{1} \mathrm{H} \mathrm{NMR}$ $\left(\mathrm{DMSO}-d_{6}\right): \delta \quad 0.79-0.89\left(18 \mathrm{H}, \mathrm{m}, \mathrm{CH}\left(\mathrm{CH}_{3}\right)_{2}\right.$ and $\left.\mathrm{CH}_{2} \mathrm{CH}_{2} \mathrm{CH}_{3}\right), 1.21-1.35$ (16H, m, aliphatic $\left.H\right), 1.89-1.96(2 \mathrm{H}$, $\left.\mathrm{m}, \mathrm{CHCH}\left(\mathrm{CH}_{3}\right)_{2}\right), 2.95\left(4 \mathrm{H}, \mathrm{q}, \mathrm{J}=6, \mathrm{NHCH}_{2} \mathrm{CH}_{2}\right), 4.17(2 \mathrm{H}$, $\mathrm{dd}, J=8.4$ and 6.6, $\mathrm{NHCHCH}), 5.31\left(2 \mathrm{H}, \mathrm{s}, \mathrm{PhCH}_{2}\right), 6.00(2 \mathrm{H}$, $\mathrm{d}, J=9$, urea $\mathrm{N} H), 6.04(2 \mathrm{H}, \mathrm{t}, J=5.6$, urea $\mathrm{N} H), 7.32-7.44$ $(5 \mathrm{H}, \mathrm{m}, \operatorname{Ar} H), 7.96(2 \mathrm{H}, \mathrm{s}, \operatorname{Ar} H), 8.24(1 \mathrm{H}, \mathrm{s}, \mathrm{Ar} H), 10.20(2 \mathrm{H}$, s, CONHAr). ${ }^{13} \mathrm{C}$ NMR (DMSO- $\left.d_{6}\right): \delta 13.9,17.9,19.3,22.1$, 26.1, 29.9, 31.0, 31.2, 39, 58.7, 66.4, 114.4, 114.8, 128.1, 128.4, 128.5, 130.3, 136.0, 139.6, 157.9, 165.4, 171.8. MS (FAB) 695 $\left(\mathrm{M}+\mathrm{H}^{+}, 12 \%\right)$. HRMS (LSIMS): calcd for $\mathrm{C}_{38} \mathrm{H}_{58} \mathrm{~N}_{6} \mathrm{O}_{6}$, 695.4491; found, 695.4501. Anal. found: $\mathrm{C}, 65.26 ; \mathrm{H}, 8.43 ; \mathrm{N}$, 11.81. $\mathrm{C}_{38} \mathrm{H}_{58} \mathrm{~N}_{6} \mathrm{O}_{6}$ requires $\mathrm{C}, 65.68 ; \mathrm{H}, 8.41 ; \mathrm{N}, 12.09$.

$2(n=6)$. The product was obtained as a white solid $(3.4 \mathrm{~g}$, $94 \%)$ from $O$-succinimidyl heptylcarbamate $6(n=6)(2.6 \mathrm{~g}$, $10.0 \mathrm{mmol}) .[\alpha]_{\mathrm{D}}=+59.7(\mathrm{c} 1.05) . \mathrm{mp} 227-228{ }^{\circ} \mathrm{C} .{ }^{1} \mathrm{H} \mathrm{NMR}$ $\left(\mathrm{DMSO}-d_{6}\right): \delta \quad 0.81-0.91\left(18 \mathrm{H}, \mathrm{m}, \mathrm{CH}\left(\mathrm{CH}_{3}\right)_{2}\right.$ and $\left.\mathrm{CH}_{2} \mathrm{CH}_{2} \mathrm{CH}_{3}\right), 1.23-1.37(20 \mathrm{H}, \mathrm{m}$, aliphatic $H), 1.90-1.97(2 \mathrm{H}$, $\left.\mathrm{m}, \mathrm{CHCH}\left(\mathrm{CH}_{3}\right)_{2}\right), 2.98\left(4 \mathrm{H}, \mathrm{q}, \mathrm{J}=6, \mathrm{NHCH}_{2} \mathrm{CH}_{2}\right), 4.18(2 \mathrm{H}$, $\mathrm{dd}, J=9.0$ and 6.6, $\mathrm{NHCHCH}), 5.34\left(2 \mathrm{H}, \mathrm{s}, \mathrm{PhCH}_{2}\right), 6.04(2 \mathrm{H}$, $\mathrm{d}, J=9.3$, urea $\mathrm{N} H), 6.07(2 \mathrm{H}, \mathrm{t}, J=5.7$, urea $\mathrm{N} H), 7.35-7.48$ $(5 \mathrm{H}, \mathrm{m}, \mathrm{Ar} H), 7.99(2 \mathrm{H}, \mathrm{s}, \operatorname{Ar} H), 8.27(1 \mathrm{H}, \mathrm{s}, \mathrm{Ar} H), 10.26(2 \mathrm{H}$, $\mathrm{s}, \mathrm{CONHAr}) .{ }^{13} \mathrm{C}$ NMR (DMSO- $\left.d_{6}\right): \delta 13.9,17.9,19.3,22.0$, 26.3, 28.4, 30.0, 31.2, 31.3, 39, 58.7, 66.3, 114.4, 114.8, 128.1, $128.4,128.5,130.3,136.0,139.6,157.9,165.3,171.8 . \mathrm{MS}$ (FAB) $723\left(\mathrm{M}+\mathrm{H}^{+}, 5 \%\right)$. HRMS (LSIMS): calcd for $\mathrm{C}_{40} \mathrm{H}_{62} \mathrm{~N}_{6} \mathrm{O}_{6}, 723.4804$; found, 723.4819. Anal. found: $\mathrm{C}$, 66.42; $\mathrm{H}, 8.68 ; \mathrm{N}, 11.63 . \mathrm{C}_{40} \mathrm{H}_{62} \mathrm{~N}_{6} \mathrm{O}_{6}$ requires $\mathrm{C}, 66.45 ; \mathrm{H}$, $8.64 ; \mathrm{N}, 11.62$.

$2(n=9)$. The product was obtained as a white solid $(3.7 \mathrm{~g}$, $92 \%)$ from $O$-succinimidyl decylcarbamate $6(n=9)(3.0 \mathrm{~g}$, $10.0 \mathrm{mmol}) .[\alpha]_{\mathrm{D}}=+53.9$ (c 1.00). mp 230-231 ${ }^{\circ} \mathrm{C} .{ }^{1} \mathrm{H}$ NMR $\left(\mathrm{DMSO}_{-} d_{6}\right): \delta \quad 0.82-0.91\left(18 \mathrm{H}, \mathrm{m}, \mathrm{CH}\left(\mathrm{CH}_{3}\right)_{2}\right.$ and $\left.\mathrm{CH}_{2} \mathrm{CH}_{2} \mathrm{CH}_{3}\right), 1.22-1.35(32 \mathrm{H}, \mathrm{m}$, aliphatic $H), 1.91-1.97(2 \mathrm{H}$, $\left.\mathrm{m}, \mathrm{CHCH}\left(\mathrm{CH}_{3}\right)_{2}\right), 2.98\left(4 \mathrm{H}, \mathrm{q}, J=6, \mathrm{NHCH}_{2} \mathrm{CH}_{2}\right), 4.18(2 \mathrm{H}$, $\mathrm{dd}, J=9.0$ and 6.6, $\mathrm{NHCHCH}), 5.34\left(2 \mathrm{H}, \mathrm{s}, \mathrm{PhCH}_{2}\right), 6.03(2 \mathrm{H}$, $\mathrm{d}, J=8.7$, urea $\mathrm{N} H), 6.06(2 \mathrm{H}, \mathrm{t}, J=3.8$, urea $\mathrm{N} H), 7.35-7.47$ $(5 \mathrm{H}, \mathrm{m}, \operatorname{Ar} H), 7.99(2 \mathrm{H}, \mathrm{s}, \operatorname{Ar} H), 8.26(1 \mathrm{H}, \mathrm{s}, \operatorname{Ar} H), 10.23(2 \mathrm{H}$, s, CONHAr). ${ }^{13} \mathrm{C}$ NMR (DMSO- $\left.d_{6}\right): \delta 13.9,17.9,19.3,22.1$, 26.4, 28.7, 28.8, 28.9, 29.0, 30.0, 31.3, 39, 58.7, 66.3, 114.4, $114.8,128.1,128.2,128.5,130.3,136.0,139.5,157.9,165.3$, 171.8. MS (FAB) $808\left(\mathrm{M}+\mathrm{H}^{+}, 5 \%\right)$. HRMS (LSIMS): calcd for $\mathrm{C}_{46} \mathrm{H}_{74} \mathrm{~N}_{6} \mathrm{O}_{6}, 807.5743$; found, 807.5730. Anal. found: $\mathrm{C}$, 67.97; $\mathrm{H}, 9.24 ; \mathrm{N}, 10.39 . \mathrm{C}_{46} \mathrm{H}_{74} \mathrm{~N}_{6} \mathrm{O}_{6}$ requires $\mathrm{C}, 68.45 ; \mathrm{H}$, $9.24 ; \mathrm{N}, 10.41$.

$2(n=10)$. The product was obtained as a white solid $(3.8 \mathrm{~g}$, $91 \%)$ from $O$-succinimidyl undecylcarbamate $6(n=10)(3.1 \mathrm{~g}$, $10.0 \mathrm{mmol}) .[\alpha]_{\mathrm{D}}=+52.3$ (c 1.02). $\mathrm{mp} 232-233{ }^{\circ} \mathrm{C} .{ }^{1} \mathrm{H} \mathrm{NMR}$
$\left(\mathrm{DMSO}-d_{6}\right): \delta 0.81-0.90\left(18 \mathrm{H}, \mathrm{m}, \mathrm{CH}\left(\mathrm{CH}_{3}\right)_{2}\right.$ and $\left.\mathrm{CH}_{2} \mathrm{CH}_{2} \mathrm{CH}_{3}\right), 1.22-1.34$ (36H, m, aliphatic $\left.H\right), 1.91-1.97(2 \mathrm{H}$, $\left.\mathrm{m}, \mathrm{CHCH}\left(\mathrm{CH}_{3}\right)_{2}\right), 2.97\left(4 \mathrm{H}, \mathrm{q}, J=6, \mathrm{NHCH}_{2} \mathrm{CH}_{2}\right), 4.18(2 \mathrm{H}$, $\mathrm{dd}, J=8.7$ and 6.6, $\mathrm{NHCHCH}), 5.34\left(2 \mathrm{H}, \mathrm{s}, \mathrm{PhCH}_{2}\right), 6.04(2 \mathrm{H}$, $\mathrm{d}, J=5.4$, urea $\mathrm{N} H), 6.07(2 \mathrm{H}, \mathrm{t}, J=5.3$, urea $\mathrm{N} H), 7.35-7.47$ $(5 \mathrm{H}, \mathrm{m}, \operatorname{Ar} H), 7.98(2 \mathrm{H}, \mathrm{s}, \operatorname{Ar} H), 8.26(1 \mathrm{H}, \mathrm{s}, \mathrm{Ar} H), 10.27(2 \mathrm{H}$, $\mathrm{s}, \mathrm{CONHAr}) .{ }^{13} \mathrm{C}$ NMR (DMSO- $\left.d_{6}\right): \delta 14.0,18.0,19.4,22.2$, 26.5, 28.8, 28.9, 29.1, 29.2, 30.1, 30.8, 31.3, 31.4, 39, 58.9, $66.5,114.6,114.9,128.2,128.3,128.7,130.4,136.1,139.7$, 158.1, 165.5, 171.9. MS (FAB) $836\left(\mathrm{M}+\mathrm{H}^{+}, 5 \%\right)$. HRMS (LSIMS): calcd for $\mathrm{C}_{48} \mathrm{H}_{78} \mathrm{~N}_{6} \mathrm{O}_{6}, 835.6056$; found, 835.6041. Anal. found: $\mathrm{C}, 68.67 ; \mathrm{H}, 9.45 ; \mathrm{N}, 10.05 . \mathrm{C}_{48} \mathrm{H}_{78} \mathrm{~N}_{6} \mathrm{O}_{6}$ requires C, 69.03; H, 9.41; N, 10.06 .

$2(n=12)$. The product was obtained as a white solid $(4.0 \mathrm{~g}$, $90 \%)$ from $O$-succinimidyl tridecylcarbamate $6(n=12)(3.4 \mathrm{~g}$, $10.0 \mathrm{mmol}) .[\alpha]_{\mathrm{D}}=+53.9$ (c 0.99). $\mathrm{mp} 235-236{ }^{\circ} \mathrm{C} .{ }^{1} \mathrm{H} \mathrm{NMR}$ $\left(\mathrm{DMSO}-d_{6}, 100{ }^{\circ} \mathrm{C}\right): \delta 0.82-0.90\left(18 \mathrm{H}, \mathrm{m}, \mathrm{CH}\left(\mathrm{CH}_{3}\right)_{2}\right.$ and $\left.\mathrm{CH}_{2} \mathrm{CH}_{2} \mathrm{CH}_{3}\right), 1.23-1.35$ (44H, m, aliphatic $\left.H\right), 1.91-1.98(2 \mathrm{H}$, $\left.\mathrm{m}, \mathrm{CHCH}\left(\mathrm{CH}_{3}\right)_{2}\right), 2.99\left(4 \mathrm{H}, \mathrm{q}, J=7, \mathrm{NHCH}_{2} \mathrm{CH}_{2}\right), 4.18(2 \mathrm{H}$, $\mathrm{dd}, J=9.0$ and 6.6, $\mathrm{NHCHCH}), 5.34\left(2 \mathrm{H}, \mathrm{s}, \mathrm{PhCH}_{2}\right), 6.02(2 \mathrm{H}$, $\mathrm{d}, J=9.6$, urea $\mathrm{N} H), 6.05(2 \mathrm{H}, \mathrm{t}, J=6.2$, urea $\mathrm{N} H), 7.35-7.47$ $(5 \mathrm{H}, \mathrm{m}, \mathrm{Ar} H), 7.98(2 \mathrm{H}, \mathrm{s}, \operatorname{Ar} H), 8.26(1 \mathrm{H}, \mathrm{s}, \mathrm{Ar} H), 10.20(2 \mathrm{H}$, $\mathrm{s}, \mathrm{CONHAr}) .{ }^{13} \mathrm{C}$ NMR (DMSO- $d_{6}, 100{ }^{\circ} \mathrm{C}$ ): $\delta 13.0,17.3,18.5$, 21.3, 25.8, 27.9, 28.1, 28.3, 29.3, 30.4, 30.6, 39, 58.7, 65.7, $114.9,115.0,127.2,127.4,127.8,130.0,135.7,138.9,157.4$, 164.9, 171.0. MS (FAB) $892\left(\mathrm{M}+\mathrm{H}^{+}, 3 \%\right)$. HRMS (LSIMS): calcd for $\mathrm{C}_{52} \mathrm{H}_{86} \mathrm{~N}_{6} \mathrm{O}_{6}, 891.6682$; found, 891.6691. Anal. found: $\mathrm{C}, 69.95 ; \mathrm{H}, 9.79 ; \mathrm{N}, 9.47 . \mathrm{C}_{52} \mathrm{H}_{86} \mathrm{~N}_{6} \mathrm{O}_{6}$ requires $\mathrm{C}$, $70.08 ; \mathrm{H}, 9.73 ; \mathrm{N}, 9.42$.

$2(n=15)$. The product was obtained as a white solid $(4.2 \mathrm{~g}$, $89 \%)$ from $O$-succinimidyl hexadecylcarbamate $6(n=15)$ (3.8 g, $10.0 \mathrm{mmol}) .[\alpha]_{\mathrm{D}}=+50.5(c 1.05) . \mathrm{mp} 239-240{ }^{\circ} \mathrm{C} .{ }^{1} \mathrm{H}$ NMR (DMSO- $\left.d_{6}, 100{ }^{\circ} \mathrm{C}\right): \delta 0.86(6 \mathrm{H}, \mathrm{t}, J=3.5$, $\left.\mathrm{CH}_{2} \mathrm{CH}_{2} \mathrm{CH}_{3}\right), 0.91\left(6 \mathrm{H}, \mathrm{d}, J=6.9, \mathrm{CH}\left(\mathrm{CH}_{3}\right) \mathrm{Me}\right), 0.94(6 \mathrm{H}, \mathrm{d}$, $\left.J=6.9, \mathrm{CHMe}\left(\mathrm{CH}_{3}\right)\right), 1.26-1.40(56 \mathrm{H}, \mathrm{m}$, aliphatic $H)$, 1.90-2.15 (2H, m, CHCH( $\left.\left(\mathrm{CH}_{3}\right)_{2}\right), 2.95-3.06(4 \mathrm{H}, \mathrm{m}$, $\left.\mathrm{NHCH}_{2} \mathrm{CH}_{2}\right), 4.19(2 \mathrm{H}, \mathrm{dd}, J=6.3$ and $9, \mathrm{NHCHCH}), 5.36$ $\left(2 \mathrm{H}, \mathrm{s}, \mathrm{PhCH}_{2}\right), 5.86(2 \mathrm{H}, \mathrm{d}, J=9$, urea $\mathrm{N} H), 5.93(2 \mathrm{H}, \mathrm{t}, J=$ 5.4, urea NH), 7.37-7.47 (5H, m, ArH), $7.95(2 \mathrm{H}, \mathrm{d}, J=2.1$, $\operatorname{Ar} H), 8.26(1 \mathrm{H}, \mathrm{t}, J=2.1, \operatorname{Ar} H), 9.90(2 \mathrm{H}, \mathrm{s}, \mathrm{CONHAr}) .{ }^{13} \mathrm{C}$ NMR (DMSO- $\left.d_{6}, 100{ }^{\circ} \mathrm{C}\right): \delta 13.0,17.3,18.6,21.3,25.8,28.0$, 28.2, 28.4, 29.4, 30.5, 30.6, 39, 58.6, 65.7, 114.87, 114.93, 127.3, 127.4, 127.9, 130.0, 135.7, 139.0, 157.5, 164.9, 171.0. MS (FAB) $976\left(\mathrm{M}+\mathrm{H}^{+}, 5 \%\right)$. HRMS (LSIMS): calcd for $\mathrm{C}_{58} \mathrm{H}_{98} \mathrm{~N}_{6} \mathrm{O}_{6}, 975.7621$; found, 975.7626. Anal. found: $\mathrm{C}$, 71.46; $\mathrm{H}, 9.94 ; \mathrm{N}, 8.35 . \mathrm{C}_{58} \mathrm{H}_{98} \mathrm{~N}_{6} \mathrm{O}_{6}$ requires $\mathrm{C}, 71.42 ; \mathrm{H}$, $10.13 ; \mathrm{N}, 8.61$. 
$2(n=18)$. The product was obtained as a white solid $(4.7 \mathrm{~g}$, $88 \%)$ from $O$-succinimidyl nonadecylcarbamate $6(n=18)(4.3$ g, $10.0 \mathrm{mmol}) .[\alpha]_{\mathrm{D}}=+52.5(\mathrm{c} 1.01) . \mathrm{mp} 243-244{ }^{\circ} \mathrm{C} .{ }^{1} \mathrm{H} \mathrm{NMR}$ $\left(\mathrm{DMSO}-d_{6}, 100{ }^{\circ} \mathrm{C}\right): \delta 0.84-0.93\left(18 \mathrm{H}, \mathrm{m}, \mathrm{CH}\left(\mathrm{CH}_{3}\right)_{2}\right.$ and $\left.\mathrm{CH}_{2} \mathrm{CH}_{2} \mathrm{CH}_{3}\right), 1.25-1.37$ (68H, m, aliphatic $\left.H\right), 1.95-1.99(2 \mathrm{H}$, $\left.\mathrm{m}, \mathrm{CHCH}\left(\mathrm{CH}_{3}\right)_{2}\right), 2.95-3.06\left(4 \mathrm{H}, \mathrm{m}, \mathrm{NHCH}_{2} \mathrm{CH}_{2}\right), 4.19(2 \mathrm{H}$, $\mathrm{dd}, J=8.4$ and 6.6, $\mathrm{NHCHCH}), 5.35\left(2 \mathrm{H}, \mathrm{s}, \mathrm{PhCH}_{2}\right), 5.92(2 \mathrm{H}$, d, $J=8.7$, urea $\mathrm{N} H), 5.98(2 \mathrm{H}, \mathrm{t}, J=5.6$, urea $\mathrm{N} H), 7.35-7.47$ $(5 \mathrm{H}, \mathrm{m}, \operatorname{Ar} H), 7.96(2 \mathrm{H}, \mathrm{s}, \operatorname{Ar} H), 8.24(1 \mathrm{H}, \mathrm{s}, \operatorname{Ar} H), 10.04(2 \mathrm{H}$, $\mathrm{s}, \mathrm{CONHAr}) .{ }^{13} \mathrm{C}$ NMR (DMSO- $\left.d_{6}, 100{ }^{\circ} \mathrm{C}\right): \delta 13.0,17.3,18.6$, 21.3, 25.8, 28.0, 28.2, 28.4, 29.4, 30.5, 30.6, 39, 58.6, 65.7, 114.87, 114.93, 127.3, 127.4, 127.9, 130.0, 135.7, 139.0, 157.5, 164.9, 171.0. MS (FAB) $1060\left(\mathrm{M}+\mathrm{H}^{+}, 3 \%\right)$. HRMS (LSIMS): calcd for $\mathrm{C}_{64} \mathrm{H}_{110} \mathrm{~N}_{6} \mathrm{O}_{6}, 1069.8560$; found, 1069.8550. Anal. found: $\mathrm{C}, 72.53 ; \mathrm{H}, 10.57 ; \mathrm{N}, 7.81 . \mathrm{C}_{64} \mathrm{H}_{110} \mathrm{~N}_{6} \mathrm{O}_{6}$ requires $\mathrm{C}$, 72.55; H, 10.46; N, 7.93 .

$2(n=20)$. The product was obtained as a white solid $(4.8 \mathrm{~g}$, $86 \%)$ from $O$-succinimidyl heneicosylcarbamate $6(n=20)(4.5$ g, $10.0 \mathrm{mmol})$. $[\alpha]_{\mathrm{D}}=+53.1$ (c 1.00). $\mathrm{mp} 249-250{ }^{\circ} \mathrm{C} .{ }^{1} \mathrm{H}$ NMR $\left(\mathrm{DMSO}-d_{6}, 100{ }^{\circ} \mathrm{C}\right): \delta 0.86\left(6 \mathrm{H}, \mathrm{t}, J=6.9, \mathrm{CH}_{2} \mathrm{CH}_{2} \mathrm{CH}_{3}\right), 0.92$ $\left(6 \mathrm{H}, \mathrm{d}, J=6.9, \mathrm{CH}\left(\mathrm{CH}_{3}\right) \mathrm{Me}\right), 0.95(6 \mathrm{H}, \mathrm{d}, J=6.9$, $\left.\mathrm{CHMe}\left(\mathrm{CH}_{3}\right)\right), 1.28-1.43(76 \mathrm{H}, \mathrm{m}$, aliphatic $H), 1.95-2.12(2 \mathrm{H}$, $\left.\mathrm{m}, \mathrm{CHCH}\left(\mathrm{CH}_{3}\right)_{2}\right), 3.00\left(4 \mathrm{H}, \mathrm{q}, J=6, \mathrm{NHCH}_{2} \mathrm{CH}_{2}\right), 4.19(2 \mathrm{H}$, $\mathrm{dd}, J=8.1$ and 6.6, $\mathrm{NHCHCH}), 5.36\left(2 \mathrm{H}, \mathrm{s}, \mathrm{PhCH}_{2}\right), 5.82(2 \mathrm{H}$, $\mathrm{d}, J=9$, urea $\mathrm{N} H), 5.88(2 \mathrm{H}, \mathrm{t}, J=5.6$, urea $\mathrm{N} H), 7.37-7.47$ $(5 \mathrm{H}, \mathrm{m}, \mathrm{Ar} H), 7.94(2 \mathrm{H}, \mathrm{d}, J=2.1, \operatorname{Ar} H), 8.19(1 \mathrm{H}, \mathrm{t}, J=1.8$, $\mathrm{Ar} H), 9.80$ (2H, s, CONHAr). ${ }^{13} \mathrm{C}$ NMR (DMSO- $\left.d_{6}, 100{ }^{\circ} \mathrm{C}\right): \delta$ $13.0,17.3,18.5,20.2,21.3,25.8,27.9,28.1,28.3,29.3,30.4$, $30.6,39,58.7,65.7,114.9,115.0,127.2,127.4,127.8,130.0$, 135.7, 138.9, 157.5, 164.9, 171.0. MS (FAB) $1116\left(\mathrm{M}+\mathrm{H}^{+}\right.$, $100 \%$ ). HRMS (LSIMS): calcd for $\mathrm{C}_{68} \mathrm{H}_{118} \mathrm{~N}_{6} \mathrm{O}_{6}, 1115.9186$; found, 1115.9192. Anal. found: $\mathrm{C}, 73.17 ; \mathrm{H}, 10.53 ; \mathrm{N}, 7.45$. $\mathrm{C}_{68} \mathrm{H}_{118} \mathrm{~N}_{6} \mathrm{O}_{6}$ requires $\mathrm{C}, 73.20 ; \mathrm{H}, 10.66 ; \mathrm{N}, 7.53$.

\section{Supporting Information}

General procedure, yields and characterization data of compounds $6(n=3-20)$.

\section{Supporting Information File 1}

Experimental Part.

[http://www.beilstein-journals.org/bjoc/content/ supplementary/1860-5397-6-114-S1.pdf]

\section{References}

1. Terech, P.; Weiss, R. G. Chem. Rev. 1997, 97, 3133-3159. doi:10.1021/cr9700282
2. van Esch, J. H.; Feringa, B. L. Angew. Chem., Int. Ed. 2000, 39, 2263-2266.

doi:10.1002/1521-3773(20000703)39:13<2263::AID-ANIE2263>3.0.CO ;2-V

3. Gronwald, O.; Snip, E.; Shinkai, S. Curr. Opin. Colloid Interface Sci. 2002, 7, 148-156. doi:10.1016/S1359-0294(02)00016-X

4. Sangeetha, N. M.; Maitra, U. Chem. Soc. Rev. 2005, 34, 821-836. doi:10.1039/b417081b

5. Dastidar, P. Chem. Soc. Rev. 2008, 37, 2699-2715. doi:10.1039/b807346e

6. Vintiloiu, A.; Leroux, J.-C. J. Controlled Release 2008, 125, 179-192. doi:10.1016/j.jconrel.2007.09.014

7. Chow, H.-F.; Zhang, J.; Lo, C.-M.; Cheung, S.-Y.; Wong, K.-W. Tetrahedron 2007, 63, 363-373. doi:10.1016/j.tet.2006.10.066

8. Chow, H.-F.; Wang, G.-X. Tetrahedron 2007, 63, 7407-7418. doi:10.1016/j.tet.2007.02.037

9. Chow, H.-F.; Zhang, J. Chem.-Eur. J. 2005, 11, 5817-5831. doi:10.1002/chem.200500174

10. Chow, H.-F.; Zhang, J. Tetrahedron 2005, 61, 11279-11290. doi:10.1016/j.tet.2005.08.006

11. Steed, J. W. Chem. Soc. Rev. 2010, 39, 3686-3699. doi:10.1039/b926219a

12. van Esch, J.; Kellogg, R. M.; Feringa, B. L. Tetrahedron Lett. 1997, 38, 281-284. doi:10.1016/S0040-4039(96)02292-7

13. van Esch, J.; de Feyter, S.; Kellogg, R. M.; de Schryver, F.; Feringa, B. L. Chem.-Eur. J. 1997, 3, 1238-1243. doi:10.1002/chem.19970030811

14. van de Laan, S.; Feringa, B. L.; Kellogg, R. M.; van Esch, J. Langmuir 2002, 18, 7136-7140. doi:10.1021/la025561d

15. George, M.; Tan, G.; John, V. T.; Weiss, R. G. Chem.-Eur. J. 2005, 11, 3243-3254. doi:10.1002/chem.200401066

16. Guichard, G.; Semetey, V.; Didierjean, C.; Aubry, A.; Briand, J. P.; Rodriguez, M. J. Org. Chem. 1999, 64, 8702-8705. doi:10.1021/jo990092e

\section{License and Terms}

This is an Open Access article under the terms of the Creative Commons Attribution License (http://creativecommons.org/licenses/by/2.0), which permits unrestricted use, distribution, and reproduction in any medium, provided the original work is properly cited.

The license is subject to the Beilstein Journal of Organic Chemistry terms and conditions:

(http://www.beilstein-journals.org/bjoc)

The definitive version of this article is the electronic one which can be found at: doi:10.3762/bjoc. 6.114 